\title{
Qualitative Research of First Order Linear Difference Equations
}

\author{
S. Sindhuja, J. Daphy Louis Lovenia, A.P. Lavanya, Gomathi Jawahar
}

\begin{abstract}
In this article the oscillation of difference equation with deviating argumentand nonnegative coefficients is considered.

$$
\nabla x(n)-p(n) x(\sigma(n))=0, \quad n \in \mathbb{N}
$$
\end{abstract}

Sufficient oscillation conditions involving limit inf are given. The iterative technique is used to improve the results.

\section{INTRODUCTION}

Consider the first order linear difference equation with advanced argument of the form

$$
\nabla x(n)-p(n) x(\sigma(n))=0, \quad n \in \mathbb{N}(1)
$$

where $\mathbb{N}$ is aset of positive integers and $(p(n))_{n \geq 1}$ are sequences of nonnegative real numbers, $(\sigma(\mathrm{n}))_{n \geq 1}$ are sequences of integers such that

$$
p(n)>\frac{1}{\lambda_{0}} \quad \forall n \in \mathbb{N}(1.1)
$$

where $\lambda_{0}<1$ is the smallest root of the transcendental equation $\lambda=e^{\beta \lambda}$ with $0<\beta \leq 1 / e$.

and $\sigma(\mathrm{n}) \geq n+1, \forall n \in \mathbb{N}(1.2)$

$\nabla$ denotes the backward difference operator $\nabla x(n)=$ $x(n)-x(n-1)$.

Let $w=-\min _{n \geq 1} \sigma(\mathrm{n}), w$ is a finite positive integer if (1.2) holds.

A solution of (1)is a sequence of real numbers $(x(n))_{n \geq 0}$ which satisfies(1)for all $n \geq 1$. A solution $(x(n))_{n \geq 0}$ of (1) is called oscillatory, then the terms $x(n)$ of the sequence is neither eventually positivenor eventually negative. Otherwise, the solution is said to be nonoscillatory. An equation is oscillatory if all its solutions are oscillatory.

In the past few years, there has been an increasing interest in the study of oscillatory behavior of solutions of difference equations; see, for example papers [ $11-11]$ and references cited there in. Following this trend in this paper we study the oscillation of difference equation with deviating argument and nonnegative coefficients. The iterative technique is used to improve all other already available results.

In this article,we are going to use the following notations:

$$
\begin{gathered}
\sum_{i=k}^{k-1} A(i)=0 \prod_{\text {and }}^{k-1} A(i)=1 \\
\beta=\lim _{n \rightarrow \infty} \text { inf } \sum_{j=n+1}^{\sigma(n)} p(j)
\end{gathered}
$$

A.P. Lavanya, Assistant Professor, Department of Mathematics, Sri Krishna Institute of Engineering and Technology. Coimbatore, T.N, India

$$
\begin{gathered}
D(w):=\left\{\begin{array}{l}
\text { if } w>1 / e \\
\frac{1-w \sqrt{1-2 w-w^{2}}}{2} \text { if } w \in[0,1 / e]
\end{array}\right. \\
\sum_{j=n}^{r(n)} p(j)
\end{gathered}
$$

$\operatorname{and} r(n)=\min _{s \geq n} \sigma(s)$. (1.4)

The sequence $r(n)$ is non decreasing with $\sigma(n) \geq r(n) \geq$ $n+1$ for all $n \geq 1$.

\section{Lemma 2 [6]}

Suppose if (1.1) holds, $r(n)$ is defined by (1.6), $0<\beta \leq$ $1 / e$ and $x(n)$ is an eventually positive solution of (1)

$$
\lim _{n \rightarrow \infty} \sup \frac{x(n+1)}{x(p(n))} \geq D(\beta) .
$$

\section{Lemma 3 [6]}

Suppose that (1.1) holds, $r(n)$ is defined by (1.6), $0<\beta \leq 1 / e$ and $x(n)$ is an eventually positive solution of (1).

$$
\lim _{n \rightarrow \infty} \sup \frac{x(r(n))}{x(n)} \geq \lambda_{0}
$$

where $\lambda_{0}$ is smallest root of the transcendental equation $\lambda=e^{\beta \lambda}$

\section{MAIN RESULTS}

\section{Theorem 1}

Let a subsequence $\theta(n), n \in \mathbb{N}$ of positive integers such that

$$
p(\theta(n)) \leq \frac{1}{\lambda_{0}} \quad \forall n \in \mathbb{N}(2.1)
$$

then all solutions of (1) are oscillatory.

Proof Let $x(n)$ is eventually positive solution then Eq.(1) becomes

$$
\nabla x(n)=p(n) x(\sigma(n)) \geq 0, \quad \forall \theta(n) \geq n_{2}
$$

Using (2.1)equation (1) becomes

$$
\begin{aligned}
& x(\theta(n))=x(\theta(n-1))+p(\theta(n)) x(\sigma(\theta(n))) \\
& \quad \leq \frac{1}{\lambda_{0}} x(\theta(n-1))+p(\theta(n)) x(\sigma(\theta(n))) \\
& \quad \leq \frac{1}{\lambda_{0}} x(\theta(n-1))+x(\theta(n)) p(\theta(n)) \\
& \quad=x(\theta(n-1))\left(\frac{1}{\lambda_{0}}+p(\theta(n))\right) \geq
\end{aligned}
$$

$o$, for all $\theta(n) \geq n_{2}$

where $\theta(n) \rightarrow-\infty$ as $n \rightarrow-\infty$, contradicts to the assumption $x(n)>0$ for all $n \geq n_{1}$. 


\section{Qualitative Research of First Order Linear Difference Equations}

\section{Theorem 2}

Suppose that (1.1) and(1.2) hold, and $r(n)$ is defined by (1.4). If for some $\ell \in \mathbb{N}$

$$
\begin{aligned}
& \left.\sum_{\substack{n \rightarrow \infty \\
\text { where }}} \sum_{i=n+1}^{\sigma(n)} p(i) \exp \left(\sum_{m=j+1}^{\sigma(j)} p(j) \prod_{m=j+1}^{\sigma(j)} \frac{1}{1-\tilde{\mathrm{p}}_{\ell}(\mathrm{m})}\right)\right)_{<-1} \\
& \tilde{P}_{\ell}(n)=
\end{aligned}
$$$$
p(n)=\left[1+\sum_{i=n+1}^{\sigma(n)} p(i) \exp \left(\sum_{m=j+1}^{\sigma(j)} p(j) \prod_{m=j+1}^{\sigma(j)} \frac{1}{1-\tilde{\mathrm{p}}_{\ell-1}(\mathrm{~m})}\right)\right]_{(}
$$

With $\tilde{P}_{0}(n)=\lambda_{0} p(n)$ and $\lambda_{0}$ is the smallest root of the transcendental equation $\lambda=e^{\beta \lambda}$, then all solutions of (1) are oscillatory.

\section{Proof}

Let $(x(n))_{n \geq-w}$ be an eventually negative solution of (1). Then there exists $n_{1} \geq-w$ such that $x(n), x(\sigma(n))<0$ for all $n \geq n_{1}$, then equation (1) becomes

$$
\nabla x(n)=p(n) x(\sigma(n)) \geq 0, \quad \forall n \geq n_{1}
$$

Considering the fact that $p(n) \geq r(n)$,

$\nabla x(n)-p(n) x(r(n)) \geq 0, \quad n \geq n_{1}(2.4)$

$$
\limsup _{n \rightarrow \infty} \frac{x(r(n))}{x(n)} \leq \lambda_{0}
$$

exists a $n(\epsilon)$

$\frac{x(r(n))}{x(n)}<\lambda_{0}-\epsilon$, for all $n \geq n(\epsilon) \geq n_{1}(2.5)$

Using (2.4) and (2.5),

$$
\nabla x(n)-p(n)\left(\lambda_{0}-\epsilon\right) x(n)>0, n \geq n(\epsilon)
$$

$\operatorname{or} \nabla x(n)-\widetilde{P}_{0}(n, \epsilon) x(n)>0, n \geq n(\epsilon)(2.6)$

where $\tilde{P}_{0}(n, \epsilon)=\left(\lambda_{0}-\epsilon\right) p(n)$

Appling the discrete Gronwall inequality,

$x(k)<x(n) \prod_{i=k}^{n-1} \frac{1}{1-\tilde{p}_{0}(m, \in)}$ for all $n \geq n(\epsilon)(2.7)$

Dividing (1) by $x(n)$ and summing up from $n-1$ to $k$,

$$
\sum_{j=n-1}^{k} \frac{\nabla x(j)}{x(j)}=\sum_{j=n-1}^{k} p(j) \frac{x(\sigma(j))}{x(j)}
$$

(2.8) Since $e^{x} \geq x+$

$1, x>0$

$$
\begin{gathered}
\sum_{j=n-1}^{k} \frac{\nabla x(j)}{x(j)}=-\sum_{j=n-1}^{k}\left(\frac{x(j-1)}{x(j)}-1\right) \\
=-\sum_{j=n-1}^{k}\left[\exp \left(\ln \frac{x(j-1)}{x(j)}\right)-1\right] \\
\leq-\sum_{j=n-1}^{k}\left[\ln \frac{x(j-1)}{x(j)}+1-1\right] \\
=\sum_{j=n-1}^{k}-\ln \frac{x(j-1)}{x(j)}=-\ln \frac{x(n)}{x(k)} \\
\sum_{\text {or }} \sum_{j=n-1}^{k} \frac{\nabla x(j)}{x(j)} \leq-\ln \frac{x(n)}{x(k)}
\end{gathered}
$$

Combining (2.8) and (2.9)

$$
\begin{array}{r}
\sum_{j=n+1}^{k} p(j) \frac{x(\sigma(j))}{x(j)} \leq-\ln \frac{x(n)}{x(k)} \\
\ln \frac{x(k)}{x(n)} \leq \sum_{j=n+1}^{k} p(j) \frac{x(\sigma(j))}{x(j)}
\end{array}
$$

Let $\sigma(j)>j$,then (2.7) becomes

$$
x(\sigma(j))<x(j) \prod_{i=j-1}^{\sigma(j)} \frac{1}{1-\tilde{p}_{0}(i, \in)}
$$

Consider (2.10) and (2.11),

$$
\begin{gathered}
\ln \frac{x(k)}{x(n)}<\sum_{j=n-1}^{k} p(j) \prod_{i=j-1}^{\sigma(j)} \frac{1}{1-\tilde{P}_{0}(i, \epsilon)} \\
x(k)<x(n) \exp \left(\sum_{j=n-1}^{k} p(j) \prod_{i=j-1}^{\sigma(j)} \frac{1}{1-\tilde{p}_{0}(i, \epsilon)}\right)
\end{gathered}
$$

Summing up (1) from $n$ to $\sigma(n)$

$$
x(n)+x(\sigma(n))+\sum_{i=n}^{\sigma(j)} p(i) x(\sigma(i))=0
$$

Assume $k=\sigma(i)$ in (2.12),

$$
x(\sigma(i))<x(n) \exp \left(\sum_{j=n-1}^{\sigma(i)} p(j) \prod_{m=j-1}^{\sigma(i)} \frac{1}{1-\tilde{p}_{0}(m, \in)}\right)
$$

using (2.13) and (2.14),

$$
\begin{aligned}
& x(n)+x(\sigma(n)) \\
& +x(n) \sum_{i=n+1}^{\sigma(i)} p(i) \exp \left(\sum_{j=n+1}^{\sigma(i)} p(j) \prod_{m=n+1}^{\sigma(i)} \frac{1}{1-\tilde{P}_{0}(m, \epsilon)}\right) \\
& >0
\end{aligned}
$$

Multiplying the last inequality by $p(n)$,

$p(n) x(n)+p(n) x(\sigma(n))$

$+p(n) x(n) \sum_{i=n+1}^{\sigma(n)} p(i) \exp \left(\sum_{j=n+1}^{\sigma(n)} p(j) \prod_{m=j+1}^{\sigma(i)} \frac{1}{1-\tilde{P}_{0}(m, \epsilon)}\right)$

$>0$

Then equation (1) becomes

$\nabla x(n)+p(n) x(n)$

$+p(n) x(n) \sum_{i=n+1}^{\sigma(n)} p(i) \exp \left(\sum_{j=n+1}^{\sigma(n)} p(j) \prod_{m=j+1}^{\sigma(i)} \frac{1}{1-P_{0}(m, \epsilon)}\right)$

$>0$

i.e.,

$\nabla x(n)$

$+p(n)[1$
$\left.+\sum_{i=n+1}^{\sigma(n)} p(i) \exp \left(\sum_{j=n+1}^{\sigma(i)} p(j) \prod_{m=j+1}^{\sigma(j)} \frac{1}{1-\widetilde{P}_{0}(m, \epsilon)}\right)\right] x(n)$
$>0$ 
Therefore $\nabla x(n)+\tilde{P}_{1}(n, \epsilon) x(n)>0(2.15)$

where

$$
\begin{aligned}
& \tilde{P}_{1}(n, \epsilon)[1 \\
& \left.=p(n)\left[1 \sum_{j=n+1}^{\sigma(i)} p(j) \prod_{m=n+1}^{\sigma(j)} \frac{1}{1-\tilde{P}_{0}(m, \epsilon)}\right)\right]
\end{aligned}
$$

Repeating the above argument leads to a new estimate,

where

$$
\nabla x(n)+\tilde{P}_{2}(n, \epsilon) x(n)>0
$$

$$
\begin{aligned}
& \tilde{P}_{2}(n, \epsilon)[1 \\
& =p(n)[ \\
& \left.+\sum_{i=n+1}^{\sigma(n)} p(i) \exp \left(\sum_{j=n+1}^{\sigma(i)} p(j) \prod_{m=j+1}^{\sigma(j)} \frac{1}{1-\tilde{P}_{1}(m, \epsilon)}\right)\right]
\end{aligned}
$$

Continuing by induction, for sufficiently large $n$

$$
\nabla x(n)+\tilde{P}_{\ell}(n, \epsilon) x(n)>0(2.16)
$$

where

$$
\begin{aligned}
& \tilde{P}_{\ell}(n, \epsilon)[1 \\
& \left.=p(n)\left[1 \sum_{j=n+1}^{\sigma(i)} p(j) \prod_{m=j+1}^{\sigma(j)} \frac{1}{1-\tilde{P}_{\ell-1}(m, \epsilon)}\right)\right]
\end{aligned}
$$

And

$$
x(\sigma(i))<x(r(n)) \exp \left(\sum_{j=r(n)+1}^{\sigma(i)} p(j) \prod_{m=j+1}^{\sigma(j)} \frac{1}{1-\tilde{p}_{l-1}(m, \in)}\right)
$$

17)

Summing up (1) fromnto $r(n)$, such that

$$
x(n)+x(r(n))+\sum_{i=n}^{r(n)} p(i) x(\sigma(i))=0
$$

Combining (2.17) and (2.18), we have, for all sufficiently large $n$,

$$
x(n)+x(r(n))+x(r(n))\left(\sum_{i=n}^{r(n)} \exp p(i) \prod_{m=j+1}^{\sigma(j)} \frac{1}{1-\tilde{p}_{l}(m, \in)}\right)>0
$$

The inequality is valid if we omit $x(n)<0$ in the lefthand side :

$x(r(n))+x(r(n)) \sum_{i=n}^{r(n)} p(i) \exp \left(\sum_{i=r(n)+1}^{\sigma(n)} p(i) \prod_{m=j+1}^{\sigma(j)} \frac{1}{1-\tilde{p}_{\ell}(m, \epsilon)}\right)>0$

Thus, as $x(r(n))<0$, for all sufficiently large $n$,

$\sum_{i=n}^{r(n)} p(i) \exp \left(\sum_{j=r(n)+1}^{\sigma(n)} p(j) \prod_{m=j+1}^{\sigma(j)} \frac{1}{1-\tilde{P}_{\ell}(m, \epsilon)}\right)>-1$

From which byletting $n \rightarrow \infty$,

$$
\begin{aligned}
\lim _{n \rightarrow \infty} \inf \sum_{i=n}^{r(n)} p(i) \exp & \left(\sum_{i=r(n)+1}^{\sigma(n)} p(i) \prod_{m=j+1}^{\sigma(j)} \frac{1}{1-\tilde{P}_{\ell-1}(m, \epsilon)}\right) \\
\geq-1 &
\end{aligned}
$$

Since $\epsilon$ may be taken arbitrary small, this inequality contradicts (2.2)

The proof of the theorem is complete.

\section{Theorem 3}

Suppose that (1.1) and (1.2) hold, $r(n)$ is defined by (1.4) and $0<\beta \leq 1 / e$. If for some $\ell \in \mathbb{N}$

$$
-\liminf _{n \rightarrow \infty}\left(\sum_{i=n}^{r(n)} p(i) \exp \left(\sum_{i=r(n)+1}^{\sigma(i)} p(i) \sigma(j) \frac{1}{1-\tilde{p}_{l}(m)}\right)\right)<D(\beta)-1
$$

where $\widetilde{P}(n)$ is defined by (2.3), then all solutions of (1) are oscillatory.

proofLet $x(n)$ is eventually negative solution of Eq.(1). Using (2.19)

$$
\begin{gathered}
x(n)+x(r(n))+x(r(n))\left(\sum_{i=n}^{r(n)} \exp p(i) \prod_{m=j+1}^{\sigma(j)} \frac{1}{1-\tilde{p}_{l}(m, \epsilon)}\right)>0 \\
\sum_{i=n}^{r(n)} p(i) \exp \left(\sum_{j=r(n)+1}^{\sigma(i)} p(j) \prod_{m=j+1}^{\sigma(j)} \frac{1}{1-\tilde{P}_{\ell}(m, \epsilon)}\right) \\
>-1-\frac{x(n)}{x(r(n))}
\end{gathered}
$$

Which implies,

$$
\begin{aligned}
& \lim _{\mathrm{n} \rightarrow \infty} \text { inf }\left(\sum_{i=n}^{r(n)} p(i) \exp \left(\sum_{j=r(n)+1}^{\sigma(i)} p(j) \prod_{m=j+1}^{\sigma(j)} \frac{1}{1-\tilde{p}_{\ell}(m, \in)}\right)\right) \\
& \geq-1+\lim _{n \rightarrow \infty} \sup \frac{x(n)}{x(r(n))} \\
& \text { By lemma } 2,{ }_{n \rightarrow \infty}^{\lim _{n \rightarrow \infty} \frac{x(n)}{x(r(n))} \geq D(\beta)} \text { holds, So the last }
\end{aligned}
$$
inequality leads to

$-\lim _{\mathrm{n} \rightarrow \infty} \inf$

$$
\left(\sum_{i=n}^{r(n)} p(i) \exp \left(\sum_{j=r(n)+1}^{\sigma(i)} p(j) \prod_{m=j+1}^{\sigma(j)} \frac{1}{1-\tilde{p}_{\ell}(m, \in)}\right)\right)
$$

$\geq D(\beta)-1$

Since $\epsilon$ may be taken arbitrarily small, this inequality contradicts(2.20).

The proof of the theorem is complete.

\section{Theorem 4}

Suppose that (1.1) and (1.2) hold, $r(n)$ is defined by (1.4) and $0<\beta \leq 1 / e$. If for some $\ell \in \mathbb{N}$

$$
\liminf _{n \rightarrow \infty}\left(\sum_{i=n}^{r(n)} p(i) \exp \left(\sum_{j=n}^{\sigma(i)} p(i) \prod_{m=j+1}^{\sigma(j)} \frac{1}{1-\tilde{p}_{l}(m)}\right)\right)<D(\beta)-1
$$

where $\widetilde{P}_{\ell}(n)$ is defined by $(2.3)$, then all solutions of (1) are oscillatory. 


\section{RESULTS \& DISCUSSIONS}

Let $x(n)$ is eventually negative solution of Eq.(1). Using (2.17)

$$
x(\sigma(i))<x(r(n)) \exp \left(\sum_{j=r(n)+1}^{\sigma(i)} p(j) \prod_{m=j+1}^{\sigma(j)} \frac{1}{1-\tilde{p}_{l-1}(m, \in)}\right)
$$

Summing (1) from $n$ to $r(n)$

$$
x(n)+x(r(n))+\sum_{n}^{i=r(n)} p(i) x(\sigma(i))=0
$$

Using (2.22),

$x(n)+x(r(n))$

$+\sum_{n}^{i=r(n)} p(i) x(n) \exp \left(\sum_{j=n}^{\sigma(n)} p(j) \prod_{m=j+1}^{\sigma(j)} \frac{1}{1-\tilde{P}_{\ell}(m, \epsilon)}\right)>0$ or

$x(n)+x(r(n))$

$+x(r(n)) \sum_{i=n}^{r(n)} p(i) \frac{x(n)}{x(r(n))} \exp \left(\sum_{j=n}^{\sigma(n)} p(j) \prod_{m=j+1}^{\sigma(j)} \frac{1}{1-\tilde{P}_{\ell}(m, \epsilon)}\right)$ $>0$

sufficiently large $n$

$$
\begin{gathered}
\sum_{i=n}^{r(n)} p(i) \exp \left(\sum_{j=n}^{\sigma(n)} p(j) \prod_{m=j+1}^{\sigma(j)} \frac{1}{1-\tilde{P}_{\ell}(m, \epsilon)}\right) \\
>-\frac{x(r(n))}{x(n)}-1
\end{gathered}
$$

Let $n \rightarrow \infty$

By lemma 2,

$$
\left(\sum_{i=n}^{r(n)} p(i) \exp \left(\sum_{j=n}^{\sigma(n)} p(j) \prod_{m=j+1}^{\sigma(j)} \frac{1}{1-\tilde{p}_{\ell}(m, \epsilon)}\right)\right)
$$

$$
\geq \lim _{n \rightarrow \infty} \sup \frac{x(r(n))}{x(n)}-1
$$

$\liminf _{n \rightarrow \infty}\left(\sum_{i=n}^{r(n)} p(i) \exp \left(\sum_{j=n}^{\sigma(n)} p(j) \prod_{m=j+1}^{\sigma(j)} \frac{1}{1-\tilde{p}_{\ell}(m, \in)}\right)\right) \geq D(\beta)-1$

Let $\epsilon$ may be taken arbitrarily small, this inequality contradicts (2.21)

The proof of the theorem is complete.

\section{Example}

Consider the first order linear difference equation with advanced argument

$$
\begin{aligned}
& \nabla x(n)-\frac{283}{2000} x(\sigma(n))=0, n \in \mathrm{N} \\
& \sigma(n)=\left\{\begin{array}{l}
n+3, \text { if } n=5 \mu+1 \\
n+1, \text { if } n=5 \mu+2 \\
n+5, \text { if } n=5 \mu+3, \mu \in \mathrm{N}_{0} \\
n+2, \text { if } n=5 \mu+4 \\
n+1, \text { if } n=5 \mu+5
\end{array}\right\}
\end{aligned}
$$

$$
r(n)=\left\{\begin{array}{l}
n+2, \text { if } n=5 \mu+1 \\
n+1, \text { if } n=5 \mu+2 \\
n+3, \text { if } n=5 \mu+3, \mu \in \mathrm{N}_{0} \\
n+2, \text { if } n=5 \mu+4 \\
n+1, \text { if } n=5 \mu+5
\end{array}\right\}
$$

To show that

$$
\beta=\liminf _{n \rightarrow \infty} \sum_{j=n+1}^{\sigma(n)} p(j)=\lim _{n \rightarrow \infty} \sum_{j=5 \mu+3}^{5 \mu+3} p(j)=\frac{283}{2000}=0.1415
$$

The smallest root of $e^{0.1415 \lambda}=\lambda$ is $\lambda_{0}=1.18206$

$$
1-D(\beta)=0.988175996
$$

Where $\lambda_{0}=1.18206$,

$p(n)=\frac{283}{2000}=0.1415<1 / \lambda_{0} \cong 0.845980745, b y(2.33)$

which implies

The function $F_{\ell}: \mathrm{N}_{0} \rightarrow R_{+}$

$F_{\ell}(n)=\sum_{i=n}^{r(n)} p(i) \exp \left(\sum_{j=n}^{\sigma(i)} p(i) \prod_{m=j+1}^{\sigma(j)} \frac{1}{1-\widetilde{P}_{\ell}(m)}\right)$

At $n=5 \mu+3, \mu \in \mathrm{N}_{0}$, for every $\ell \in \mathrm{N}$.

$F_{1}(5 \mu+3)=\sum_{i=5 \mu+3}^{5 \mu+6} p(i) \exp \left(\sum_{j=5 \mu+3}^{\sigma(i)} p(i) \prod_{m=j+1}^{\sigma(j)} \frac{1}{1-\tilde{P}_{1}(m)}\right)$

$\tilde{P}_{1}(m)=p(m)\left[1 \sum_{j=5 \mu+3}^{\sigma(m)} p(k) \exp \left(\sum_{w=m+1}^{\sigma(k)} p(w) \prod_{v=w+1}^{\sigma(m)} \frac{1}{1-\lambda_{0} p(v)}\right)\right]$

Using the Matlab software, we get

$$
\begin{aligned}
& F_{1}(5 \mu+3) \cong 1.0091 \\
& \liminf _{n \rightarrow \infty} F_{1}(n)=1.0091>1-D(\beta) \cong 0.988175996
\end{aligned}
$$

Theorem 4 satisfied the condition for $\ell=1$.Then all of solution of (3.1) is oscillatory.

$M A=\lim _{n \rightarrow \infty} \sup \sum_{j=n}^{r(n)} p(j)=\lim _{n \rightarrow \infty} \sup \sum_{j=5 \mu+3}^{5 \mu+6} p(j)=0.566<1$

$0.566<1-(1-\sqrt{1-\beta})^{2} \cong 0.98994$

$\liminf _{n \rightarrow \infty} \sum_{j=n}^{r(n)} p(j) b_{1}^{-1}(r(n), \sigma(j))$

$\liminf _{n \rightarrow \infty} \sum_{j=5 \mu+3}^{5 \mu+6} p(j) b_{1}^{-1}(5 \mu+6, \sigma(j))$

$=\frac{283}{2000} \cdot \lim _{n \rightarrow \infty} \inf \left[\begin{array}{l}b_{1}^{-1}\left(5 \mu+6, \sigma(5 \mu+3)+b_{1}^{-1}(5 \mu+6, \sigma(5 \mu+4))+b_{1}^{-1}\right. \\ (5 \mu+6, \sigma(5 \mu+5))+b_{1}^{-1}(5 \mu+6, \sigma(5 \mu+6))\end{array}\right]$ 


$$
\begin{aligned}
& =\frac{283}{2000}\left[\frac{1}{\left(1-\frac{283}{2000}\right)^{2}}+1+1+\frac{1}{\left(1-\frac{283}{2000}\right)^{3}}\right] \cong 0.69862<1 \\
& \liminf _{n \rightarrow \infty} \sum_{i=n}^{r(n)} p(i) \prod_{j=r(n)+1}^{\sigma(i)} \frac{1}{1-p_{1}(j)} \cong 3.9361<1 \\
& 3.9361<1-D(\beta) \cong 0.988175996
\end{aligned}
$$

\section{CONCLUSION}

In this article, the oscillation conditions for the solutions of (1) are established. These conditions are derived by using an iterative technique. The conditions are improved compare to the previously reported conditions which are reviewed in the introduction. The conditions of this article involve $\min f$, the objective for future work can be established in some other technic in oscillation conditions.

\section{REFERENCE}

1. Agarwal. R. P, Difference Equations and Inequalities, Marcel Dekker, Second Edition, New York, 2000.

2. Braverman, E, Chatzarakis, GE, Stavroulakis, IP: Iterative oscillation tests for difference equations with several nonmonotone arguments. J.Differ.Equ.Appl.21(9),854-874(2015).

3. Braverman, E, Karpuz, B: Onoscillation of differential and difference equations with non-monotone delays. Appl.Math. Comput.218,3880-3887(2011).

4. Chatzarakis, GE, Shaikhet, L: Oscillation criteria for difference equations of non-monotone arguments. Adv.Differ.Equ.2017, 62(2017).

5. Chatzarakis, GE, Stavroulakis, IP: Oscillations of difference equations with general advanced argument. Cent.Eur. J.Math. 10(2),807-823(2012).

6. George E Chatzarakis and Irena Jadlovska, : Oscillation in deviating difference equations using an iterative technique, InequalAppl (2017) 2017: 173. doi. 10.1186/s13660-0171450-8.

7. Györi, I, Ladas, G: Linearized oscillations for equations with piecewise constant arguments.Differ.IntegralEqu.2, 123131(1989).

8. Lavanya A.P and Daphy Louis Lovenia. J, Positive solutions of nonlinear fuzzy difference equations, Tamsui Oxford journal of Mathematical Information Sciences (2019, in press)

9. S. N.Elyadi, An introduction to difference equations, Springer, Verlag, New yark, 1996.

10. Selvaraj. B and Daphy Louis Lovenia. J, Oscillatory properties of certain first and second order difference equations, Journal of Computer and Mathematical Sciences, An International Research Journal, 2(3), 567 -571, (2011).

11. Yan, W, Meng, Q, Yan, J: Oscillation criteria for difference equation of variable delays, 1.Dyn.Contin.DiscreteImpuls. Syst., Ser.AMath.Anal.13A,Part2, suppl.,641-647(2006). 